\title{
Learning to Control Arm Stiffness Under Static Conditions
}

\author{
Mohammad Darainy, ${ }^{1,2}$ Nicole Malfait, ${ }^{2}$ Paul L. Gribble, ${ }^{3}$ Farzad Towhidkhah, ${ }^{1}$ and David J. Ostry ${ }^{2,4}$ \\ ${ }^{1}$ Department of Biomedical Engineering, AmirKabir University of Technology, Tehran 15875-4413, Iran; ${ }^{2}$ Department of Psychology, \\ McGill University, Montreal H3A 1B1; ${ }^{3}$ Department of Psychology, The University of Western Ontario, London, N6A 5C2, Canada; and \\ ${ }^{4}$ Haskins Laboratories, New Haven, Connecticut 06511
}

Submitted 10 June 2004; accepted in final form 22 July 2004

\begin{abstract}
Darainy, Mohammad, Nicole Malfait, Paul L. Gribble, Farzad Towhidkhah, and David J. Ostry. Learning to control arm stiffness under static conditions. J Neurophysiol 92: 3344-3350, 2004. First published July 28, 2004; doi:10.1152/jn.00596.2004. We used a robotic device to test the idea that impedance control involves a process of learning or adaptation that is acquired over time and permits the voluntary control of the pattern of stiffness at the hand. The tests were conducted in statics. Subjects were trained over the course of 3 successive days to resist the effects of one of three different kinds of mechanical loads: single axis loads acting in the lateral direction, single axis loads acting in the forward/backward direction, and isotropic loads that perturbed the limb in eight directions about a circle. We found that subjects in contact with single axis loads voluntarily modified their hand stiffness orientation such that changes to the direction of maximum stiffness mirrored the direction of applied load. In the case of isotropic loads, a uniform increase in endpoint stiffness was observed. Using a physiologically realistic model of two-joint arm movement, the experimentally determined pattern of impedance change could be replicated by assuming that coactivation of elbow and double joint muscles was independent of coactivation of muscles at the shoulder. Moreover, using this pattern of coactivation control we were able to replicate an asymmetric pattern of rotation of the stiffness ellipse that was observed empirically. These findings are consistent with the idea that arm stiffness is controlled through the use of at least two independent co-contraction commands.
\end{abstract}

\section{IN T R O D U C T I O N}

The idea that the nervous system can modify the mechanical behavior of the neuromuscular periphery (Hogan 1985), known as impedance control, has been a focus of interest in recent studies of motor control. The appeal of the idea is that impedance control might provide a means to ensure stability during interactions with the environment. A number of studies have examined muscle co-contraction in single joint movement in the context of unstable mechanical loads (e.g., De Serres and Milner 1991; Milner 2002a). In work on two-joint movement, evidence that subjects can alter the pattern of arm impedance has been reported in studies that have assessed stiffness during movement (Burdet et al. 2001; Franklin et al. 2003) and in studies in which the limb is stationary but when the subject is actively applying force (Franklin and Milner 2003; Gomi and Osu 1998; McIntyre et al. 1996; Perreault et al. 2002). However, in the two-joint arm, little is known about the control of impedance when the limb is stationary and net force output is zero. This study focuses on the control of limb impedance under conditions in which the limb is in static equilibrium. We test the idea that impedance control involves a process of

Address for reprint requests and other correspondence: D. J. Ostry, Dept. of Psychology, McGill Univ., 1205 Dr. Penfield Ave., Montreal, Quebec H3A 1B1, Canada (E-mail: ostry@motion.psych.mcgill.ca). learning or adaptation, as has been reported for other predictive adjustments that are involved in compensation for mechanical loads (Lackner and DiZio 1994; Sainburg et al. 1999; Shadmehr and Mussa-Ivaldi 1994).

There are good reasons to focus on the capacity for voluntary modification of static arm stiffness. Unlike measures of stiffness during movement or during active force production that are affected by phasic muscle activity, stiffness in statics is primarily dependent on antagonist muscle coactivation and afferent feedback and hence reflects the control that acts to stabilize the limb (limb geometry and muscle mechanical properties play a role in all measures of stiffness). Measures of stiffness during movement can be difficult to interpret because the resistance to displacement is partly a bi-product of the phasic signals that are associated with the production of movements. The evaluation of stiffness under static conditions eliminates this confound and permits a direct examination of one of the limits of voluntary stiffness control.

In initial reports of human arm stiffness in a horizontal plane, Mussa-Ivaldi et al. (1985) documented the spring-like properties of muscles acting about the shoulder and elbow. The pattern of stiffness at the hand was depicted as an ellipse that had a major axis that corresponded to direction of maximum stiffness. It was suggested that the size of the ellipse, and hence the overall stiffness magnitude, could be changed by cocontracting shoulder and elbow muscles.

There is, in fact, little evidence that the pattern of stiffness under static conditions is modifiable, when the limb is stationary and net force is zero. Work by Mussa-Ivaldi et al. (1985) favored the idea that the control of impedance was restricted to rather global adjustments of the impedance of the limb as a whole. Gribble and Ostry (1998) report independent control of shoulder and elbow stiffness. However, the claim is based on electromyographic data and is thus indirect. Lacquaniti et al. (1993) concluded that there was voluntary modification of stiffness during the preparation phase of ball catching. In the Lacquaniti et al. data, the largest changes in stiffness occur subsequent to catching rather than preceding it. Thus changes in impedance may be as much a reaction to contact with the ball as an adjustment that occurs in anticipation of the load. Voluntary changes to the stiffness ellipse at the hand have also been reported in the context of isometric force production (Franklin and Milner 2003; Gomi and Osu 1998; McIntyre et al. 1996; Perreault et al. 2002). However, under these conditions, measures of stiffness are contaminated by active force output and thus measured stiffness reflects both the neural

\footnotetext{
The costs of publication of this article were defrayed in part by the payment of page charges. The article must therefore be hereby marked "advertisement" in accordance with 18 U.S.C. Section 1734 solely to indicate this fact.
} 
control of impedance and changes in impedance due to force generation. Indeed, the only indication to date that the pattern of impedance may be voluntarily modified when the limb is stationary has involved biofeedback procedures in which subjects were able to modify impedance based on visual displays of either EMG activity (Gomi and Osu 1998) or static endpoint stiffness (Perreault et al. 2002). Even in these situations, it remains uncertain whether changes in endpoint impedance can be achieved using only the kinaesthetic information that would ordinarily accompany limb movement and placement.

In this study, we have applied mechanical loads to the arm to assess the extent to which subjects can modify the pattern of impedance at the hand. Training took place over 3 consecutive days with the goal of examining the way in which the pattern of impedance develops as a function of time. We showed that, depending on the direction of the destabilizing load, subjects gradually modify the orientation of maximum stiffness at the hand over the course of training. Moreover, using a physiologically realistic model of the two-joint arm, we suggest that the observed changes arise as a consequence of independent coactivation of muscles at the elbow and the shoulder.

\section{METHODS}

\section{Experimental setup}

Twenty-four right-handed subjects, between the ages of 20 and 32, took part in this study. The subjects were seated in front of a two degree-of-freedom robotic arm (Interactive Motion Technologies, Cambridge, MA). Position measurements were obtained using 16-bit encoders (Gurley Precision Instruments). Forces were measured with a force-torque sensor (ATI Industrial Automation) that was mounted above the handle of the manipulandum. Shoulder movement was restricted by a harness and the wrist was restrained by a wrist brace (see Fig. 1A). Subjects gripped the handle of the manipulandum; their right arm was supported against gravity by an air sled. Subjects were placed in a standard position relative to the robot such that the angle at the elbow was $90^{\circ}$ and the angle at the shoulder was $45^{\circ}$ (relative to the frontal plane). The shoulder was abducted to $70^{\circ}$. A computer monitor was positioned next to the robotic arm and in front of the subjects. A 20-mm red circle in the middle of the screen specified the
A

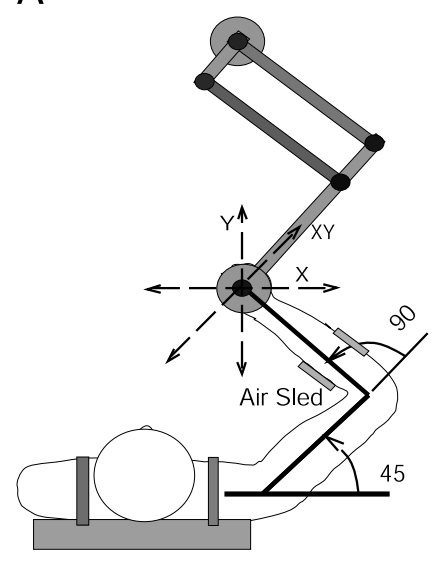

B

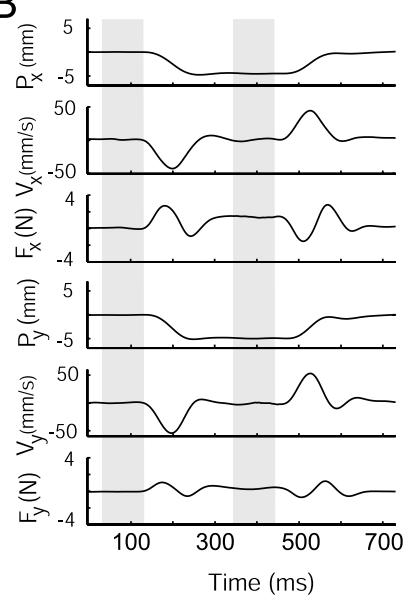

FIG. 1. A: experimental setup showing directions of force application. $B$ : representative example of servo-position measurement. $P_{\mathrm{x}}$ and $P_{\mathrm{y}}$ give the position of the hand, $V_{\mathrm{x}}$ and $V_{\mathrm{y}}$, velocities, $F_{\mathrm{x}}$ and $F_{\mathrm{y}}$, sensed forces. Highlighted bars indicate the data used to measure position change and restoring force. target location. A 15-mm yellow circle indicated the position of the hand. The subject's arm was visible throughout the procedure.

\section{Null field task}

The initial phase of the experiment (the null-field condition) involved the estimation of stiffness in the absence of any other manipulation. Subjects were required to place a yellow circle that corresponded to the position of the hand in the middle of the target zone and remain on target. Subjects were instructed not to intervene in any manner during this phase of the experiment.

To estimate stiffness, we used a method introduced by Mussa-Ivaldi et al. (1985). The hand was displaced using a position servo in each of eight equally spaced directions about a circle, and measures of displacement and restoring force were obtained. The gain of the servo was $4,000 \mathrm{~N} / \mathrm{m}$. Visual feedback of endpoint position was frozen on the computer screen during this time. The displacements occurred in random order. They had a commanded amplitude of $7 \mathrm{~mm}$ [actual amplitudes averaged $6.1 \pm 0.5(\mathrm{SD}) \mathrm{mm}$ ], a hold time of either 200 (12 subjects) or $400 \mathrm{~ms}$ (12 subjects), and were ramped on and off over a 100-ms interval (Fig. 1B). Prior to each displacement, the subject's hand had to be within the target zone, and the hand velocity had to be $<1 \mathrm{~mm} / \mathrm{s}$. Once these conditions were satisfied, a measurement was initiated at a randomly selected time between 1.5 and $2.5 \mathrm{~s}$.

\section{Postural maintenance during force application}

The null-field measurements were followed by a series of trials that were designed to produce a selective increase in stiffness. For this task, subjects were randomly assigned to one of three groups that trained to resist different patterns of load. Subjects were trained in a single experimental condition over 3 consecutive days. The task involved the application of single-axis force pulses either in the lateral direction (Fig. 1, $x$-axis), the forward/backward direction ( $y$-axis), or the application of a uniform pattern of loads in which force pulses were delivered in one of eight directions about a circle (isotropic condition). Subjects were instructed to maintain the position of the limb in the middle of the target and were told nothing whatsoever about the characteristics of the load. In the absence of predictive adjustments in stiffness on the part of the subject, the load was sufficient to move the arm from the target zone. At the end of each experimental block, the total time that the hand of the subject was outside of the target was displayed as feedback. Subjects were asked to try to reduce this value as much as possible while avoiding excessive co-contraction to reduce the likelihood of fatigue.

The timing of the perturbations and their specific directions were unpredictable. For subjects trained with loads in the $x$ - and $y$-directions, the actual orientation of force application along the specified axis was chosen randomly. For the third group, forces were applied in each of eight directions in a random order (isotropic field). The applied forces were $2 \mathrm{~N}$ in magnitude and had a 300-ms hold time. The time interval between forces was selected randomly between 0.5 and $1.5 \mathrm{~s}$.

Each day of the experiment, subjects completed nine blocks of 40 perturbations each. Servo-controlled position displacements, equivalent to those used to estimate stiffness in null field conditions, were randomly interspersed within each of the nine blocks of trials. Stiffness matrices were computed based on data pooled over each three blocks. Thus three such estimates of stiffness were obtained for each subject on each day of testing.

We approached the issue of voluntary intervention in two ways. We first eliminated those trials in which the variability of velocity during the hold phase of movement exceeded $4 \mathrm{~mm} / \mathrm{s}$. For the remainder of the data set, we computed on a trial-by-trial basis the maximum change in position, velocity and force relative to the average value obtained during the hold phase of measurement displacement. Thus first, to ensure that the subject's hand was stationary, the SD of hand 
velocities was computed in a 100-ms interval before the initiation of the servo-displacement and during the last $100 \mathrm{~ms}$ of the hold phase. If the SD based on a pooled estimate of variance was $>4 \mathrm{~mm} / \mathrm{s}$, the trial was dropped from analysis. This resulted in the rejection of $2.9 \%$ of the data.

In the data that were used for analysis, maximum changes in hand position, velocity, and restoring force were computed in each interval used for the estimation of stiffness and averaged over subjects, days, and displacement directions. The average maximum for position change was $0.12 \mathrm{~mm}$, for velocity change, $2.1 \mathrm{~mm} / \mathrm{s}$, and for force change, 0.19 N. Similar values were obtained with the 200- and 400-ms hold times. These values reassured us that voluntary intervention was unlikely in the data used for stiffness estimation.

\section{Data analysis}

Hand positions and forces were sampled at $200 \mathrm{~Hz}$. The position and force signals were low-pass Butterworth-filtered with a secondorder zero phase-lag filter at $40 \mathrm{~Hz}$. Position signals were numerically differentiated to produce velocity estimates. The relationship between imposed displacements and resulting forces can be written as

$$
\left[\begin{array}{l}
d f_{\mathrm{x}} \\
d f_{\mathrm{y}}
\end{array}\right]=\left[\begin{array}{ll}
K_{\mathrm{xx}} & K_{\mathrm{xy}} \\
K_{\mathrm{yx}} & K_{\mathrm{yy}}
\end{array}\right]\left[\begin{array}{l}
d x \\
d y
\end{array}\right]
$$

To estimate the stiffness matrix $K$, we used three displacements in each of eight randomized directions and measured the associated restoring forces. Differences in the mean value of position between the $100-\mathrm{ms}$ interval prior to the perturbation and the $100-\mathrm{ms}$ interval at the end of the hold phase gave values for $d x$ and $d y$. Mean force estimates during the same intervals provided values for $d f x$ and $d f y$. Linear regression was used to estimate the stiffness matrix.

In purely elastic systems, the off-diagonal terms of the stiffness matrix $\left(K_{\mathrm{xy}}\right.$ and $\left.K_{\mathrm{yx}}\right)$ are equal in magnitude. Following Mussa-Ivaldi et al. (1985), estimates of stiffness were decomposed into symmetric $\left(K_{\mathrm{s}}\right)$ and antisymmetric terms $\left(K_{\mathrm{a}}\right)$ where

$$
\begin{aligned}
& K_{\mathrm{s}}=\left\lfloor\begin{array}{cc}
K_{\mathrm{xx}} & 1 / 2\left(K_{\mathrm{xy}}+K_{\mathrm{yx}}\right) \\
1 / 2\left(K_{\mathrm{xy}}-K_{\mathrm{yx}}\right) & K_{\mathrm{yy}}
\end{array}\right\rfloor \\
& K_{\mathrm{a}}=\left\lfloor\begin{array}{cc}
0 & 1 / 2\left(K_{\mathrm{xy}}+K_{\mathrm{yx}}\right) \\
1 / 2\left(K_{\mathrm{xy}}-K_{\mathrm{yx}}\right) & 0
\end{array}\right\rfloor
\end{aligned}
$$

and $K=K_{\mathrm{s}}+K_{a}$. The proportion of variance accounted for by the symmetric term is given by

$$
R^{2}=1-\frac{\sum\left(F-F^{\prime}\right)^{2}}{\sum(F-\bar{F})^{2}}
$$

where $F$ is a matrix of measured forces, $F^{\prime}$ gives values predicted using the symmetric matrix $\left(K_{\mathrm{s}}\right)$, and $F$ is the mean of measured forces. The summation is applied over all directions of force application. It was found that the proportion of variance accounted for by the symmetric term ranged from 0.58 to 0.97 across subjects, with a mean of 0.87 . In $94.5 \%$ of cases, $R^{2}>0.7$. Thus, in this study, the arm is dominated by its spring-like behavior.

Following Mussa-Ivaldi et al. (1985), the pattern of stiffness can be visualized as an ellipse in which the restoring force is shown for all directions in horizontal plane. Singular value decomposition of the stiffness matrix provides values for the orientation, shape, and size of the ellipse.

\section{RES ULTS}

\section{Effect of learning on hand endpoint stiffness}

The aim of this study was to assess the extent to which the pattern of endpoint stiffness could be modified over the course of learning in a task that required postural maintenance. Stiffness was first estimated for all subjects under null-field conditions on each day of training. There were no reliable changes in the orientation of the null-field stiffness ellipse over training or as a function of the direction of load application $(P>0.05)$. The mean orientation of the null field ellipse was $124.6 \pm 8.7^{\circ}$ relative to the frontal plane.

Figure 2 shows, under conditions of load application, typical changes for individual subjects in the stiffness ellipse over the course of 3 consecutive days of training. It may be seen that over days, the orientation of the stiffness ellipse gradually changes toward the direction of the externally applied load for subjects trained with forces in the $x$ - and $y$-directions. For subjects trained using an isotropic pattern of forces, stiffness magnitudes were high, and there was no change in the orientation of the stiffness ellipse.

Figure 3 shows mean values of stiffness change averaged over subjects for each day of training. The orientation and size of the ellipse as well as the time outside of the target zone are presented separately. Over days, the stiffness ellipses rotated clockwise for subjects tested with perturbations in the $y$ direction, whereas for subjects tested in the $x$-direction, the ellipses rotated counterclockwise (Fig. 3A). The change in orientation over days was assessed using repeated measures ANOVA. Posthoc comparisons were carried out using Bonferroni-Holm tests. For forces applied in both $x$ - and $y$-directions, the orientation on day 3 was reliably different from the orientation on day 1 ( $P<0.01$ for $y$ and $P<0.05$ for $x$ ), indicating that subjects learned to voluntarily change the pattern of hand stiffness to increase resistance in the direction of load. For the $x$-direction, where the magnitude of the effects was smaller, the change in orientation relative to the null field was reliably different from zero only on days 2 and 3 of training $(P<0.01$

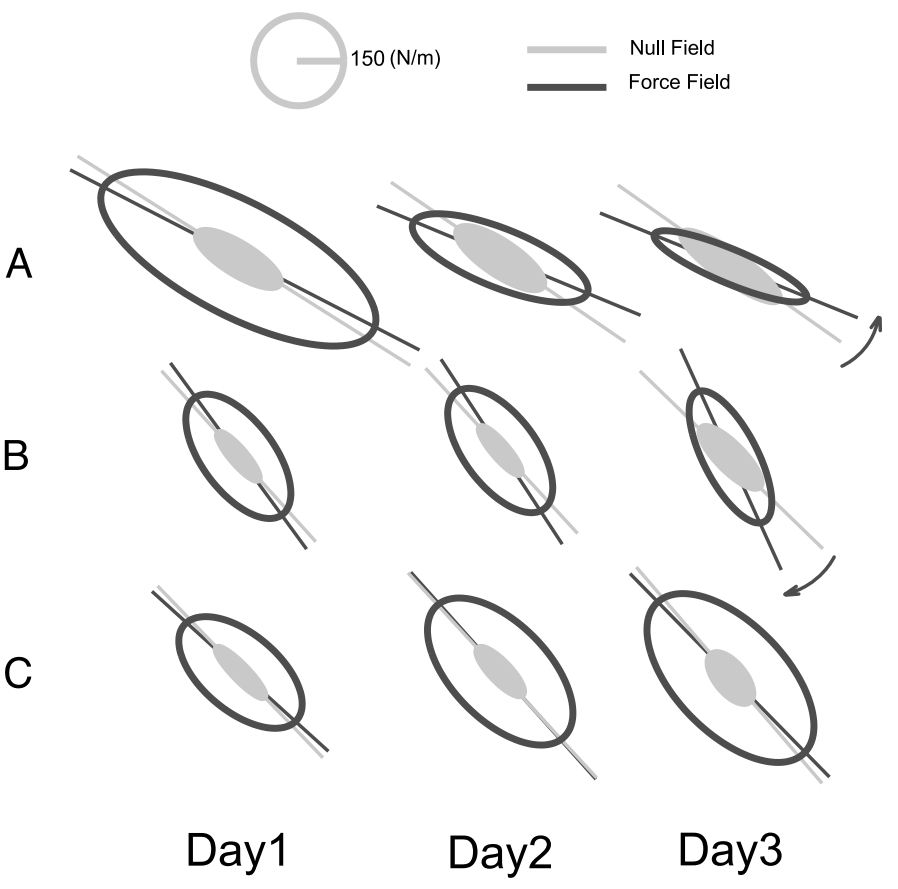

FIG. 2. Hand stiffness ellipses for 3 subjects over the course of 3 days of training. $A$ : subject trained to resist loads along a lateral axis ( $x$-direction). $B$ : subject trained to resist a forward/backward load ( $y$-direction). $C$ : subject trained using isotropic force-field. 

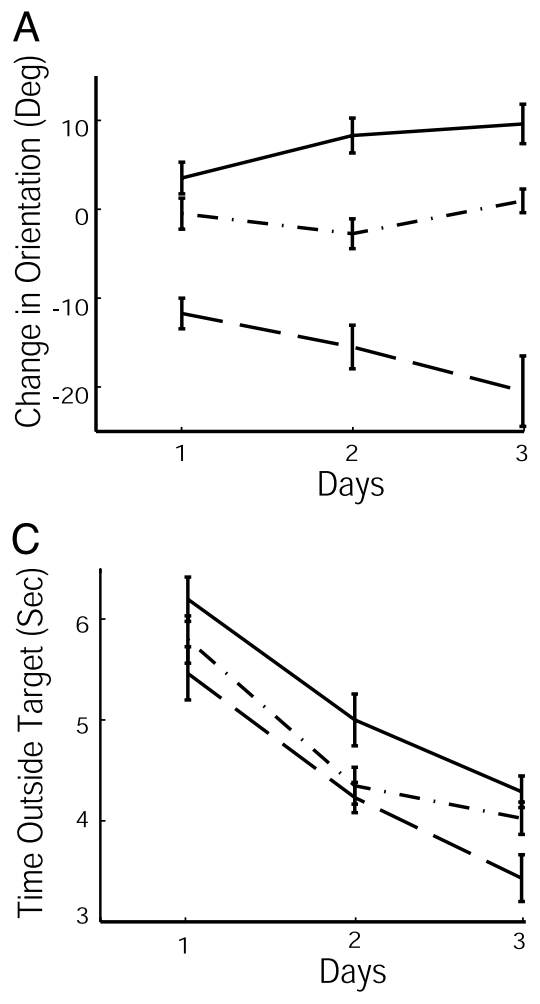

in both cases), which points to the gradual nature of impedance learning over time. When force application was isotropic, there was no reliable change in orientation $(P>0.05)$. It may also be seen that, despite the large number of training trials (864 trials/subject), adaptation to the direction of external load was incomplete. For subjects trained in the $y$-direction, the average change in orientation of the stiffness at the end of training was $21.35^{\circ}$. For subjects trained in the $x$-direction, stiffness orientation changed on average by $9.45^{\circ}$. Note as well that the ability to compensate for loads in $x$ and $y$ does not reflect an initial orientation (null-field orientation) that is closer to one perturbation direction than the other. In fact, null field stiffness is closer to $y$ than to $x$, but changes in $y$ are nevertheless greater.

Figure $3 B$ shows the size (enclosed area) of the stiffness ellipse for subjects trained with forces in $x$ and $y$ and also for subjects in the isotropic condition. The size of the ellipse for the isotropic environment was reliably greater than for the two other conditions $(P<0.01)$. In all three conditions, the size of the ellipse was reliably greater than in the absence of load $(P<$ $0.01)$. Thus, whereas subjects in isotropic condition scaled up the magnitude of hand endpoint stiffness, subjects trained with loads in the $x$ - and $y$-directions adapt by rotating their stiffness ellipse in the direction of the external load and by increasing its size. In addition, there is substantially greater variability in size in the isotropic condition, indicating that subjects adopt quite different strategies in trying to adapt to the field under these load conditions.

The shape of the stiffness ellipse was defined as the ratio of the major to the minor axis. Change in shape due to the application of load was quantified on a per subject basis. We found no reliable differences in shape as result of the different experimental conditions or over days ( $P>0.05$ in both cases).

Figure $3 D$ shows the total time that the subject's hand was outside of the target zone over the course of training. It can be seen that all three groups successfully learned to do the task as reflected by the fact that they were able to stay longer on target over the period of training ( $P<0.01$ for all groups).

We assessed the extent to which the changes in impedance patterns were anticipatory by examining the mean restoring force in different $100-\mathrm{ms}$ intervals within the hold phase. If changes in stiffness were a reaction to load application, one might expect that restoring force would increase over the hold phase of the measurement displacement. For the 200-ms hold phase, the mean restoring force in the two 100-ms intervals was 3.68 and $3.71 \mathrm{~N}$, respectively. For the 400-ms hold phase, the mean restoring force actually decreased. Mean values were 3.49, 3.43, 3.42 , and $3.31 \mathrm{~N}$ from the start to the end of the hold phase.

\section{Simulation studies}

To better understand the empirically observed patterns of hand stiffness, we simulated the changes in stiffness that might be expected through the control of muscle coactivation using a two-joint planar arm model described in Gribble et al. (1998).

Modeled control signals were based on the $\lambda$ version of equilibrium point hypothesis. Commands analogous to the $\mathrm{R}$ and $\mathrm{C}$ commands in previous versions of the model (Feldman et al. 1990) were used to produce rotation of the joints and, independently, muscle co-activation without movement. In these simulations, values associated with the $\mathrm{R}$ command were held constant to simulate postural maintenance, while values for the $\mathrm{C}$ command were varied to produce changes in the level of muscle co-activation. Consistent with experimental evidence that subjects can independently co-activate muscles at the shoulder and elbow (Gomi and Osu 1998; Gribble and Ostry 1998), separate co-contraction commands were defined for each of these joints. The elbow co-activation command was applied to single joint elbow muscles and biarticular muscles 
(Gomi and Osu 1998; Gribble and Ostry 1998). The shoulder co-contraction command was applied to single joint shoulder muscles. The commands enabled changes in co-activation at either the elbow or shoulder that were independent of cocontraction at the other joint.

Elbow and shoulder co-activation commands in the model were both initially set to $10 \mathrm{~N}$ to match the empirical pattern of null field stiffness. That is, using muscle $\lambda \mathrm{s}$ that produce a total muscle force of $10 \mathrm{~N}$ at each of the shoulder and elbow, patterns of simulated stiffness were similar to those observed empirically. To assess the effects of differentially modifying co-contraction at the shoulder and the elbow, we held constant the modeled co-contraction command to one joint and systematically changed co-contraction command to the other joint from 10 to $120 \mathrm{~N}$. Using the different combinations of shoulder and elbow co-contraction, we computed the endpoint stiffness that would result using a simulated displacement that was comparable to that used experimentally.

Figure 4, $A$ and $B$, shows the results of simulations. In Fig. $4 A$, the solid line gives the orientation of the stiffness ellipse for conditions in which shoulder contraction was varied from
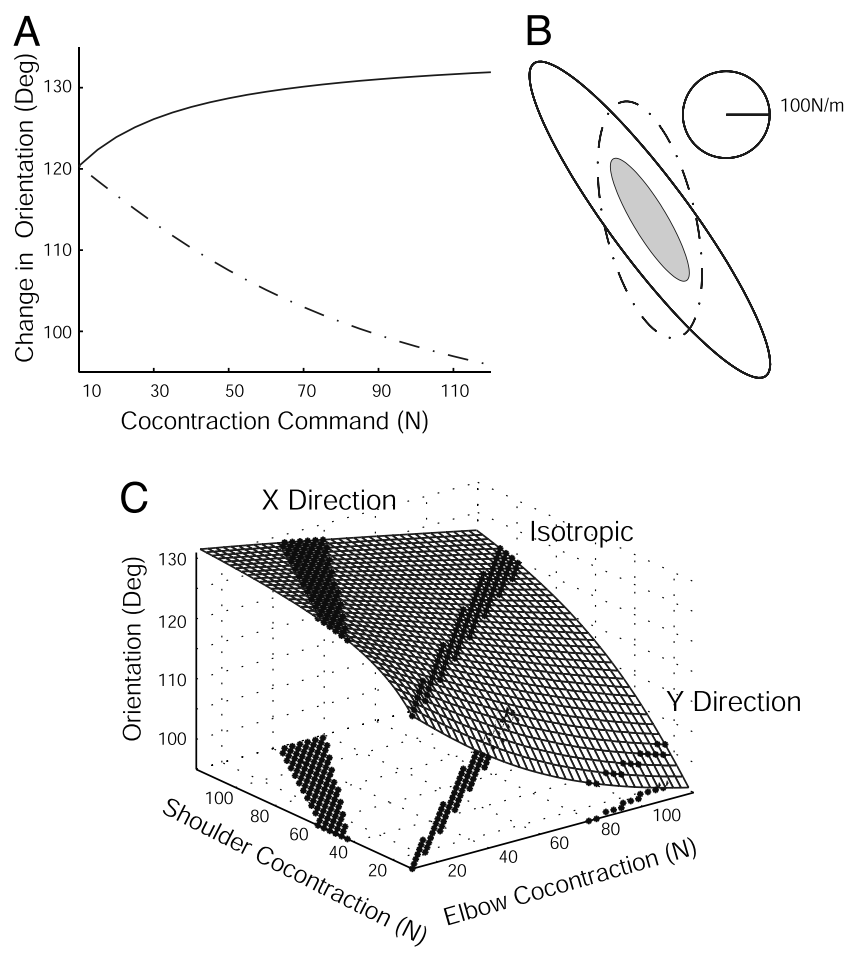

FIG. 4. Modeling results showing the effects of different co-contraction levels on stiffness. A: changes in ellipse orientation with differences in co-contraction command. Solid line shows predicted changes in hand stiffness orientation when the co-contraction to the elbow is constant and the command to the shoulder is varied. Dashed lines show changes in predicted changes to the ellipse orientation when the shoulder co-contraction is constant and the elbow is varied. $B$ : predicted changes in the orientation of the hand stiffness ellipse. Ellipse shown with solid lines uses elbow and shoulder co-contraction levels of 20 and $80 \mathrm{~N}$, respectively. Ellipse in dashed lines uses elbow and shoulder commands of 100 and $10 \mathrm{~N}$, respectively. Smaller null field ellipse uses equal magnitude elbow and shoulder commands ( $10 \mathrm{~N}$ in each case). $C$ : predicted stiffness orientation as a function of different co-contraction levels at the elbow and shoulder. Darkened bands on the upper surface show the range of empirically observed stiffness orientations for subjects tested with loads in $x, y$, and isotropic forces. The projection onto the co-contraction plane gives the range of modeled co-contraction levels that correspond to the empirically obtained results.
10 to $120 \mathrm{~N}$ and elbow co-contraction is constant $(10 \mathrm{~N})$. The dashed line shows ellipse orientation when elbow co-contraction varied $(10-120 \mathrm{~N})$, and shoulder co-contraction was constant $(10 \mathrm{~N})$. It can be seen that when the co-contraction at the elbow is increased, the simulated stiffness ellipse rotates in the direction of the $y$-axis. When co-contraction at the shoulder is increased, the ellipse rotates in the direction of the $x$-axis. The changes in orientation that result from independent coactivation of elbow and shoulder muscles are thus consistent with the empirically observed changes described above. It may also be seen that the orientation of the stiffness ellipse has a nonlinear relation to the simulated co-contraction level. When shoulder co-contraction is varied and the command to the elbow is fixed, changes in the ellipse orientation quickly reach a limit. A similar pattern is observed when elbow co-contraction is varied and the command to the shoulder is fixed.

The magnitudes of orientation change in the simulations were comparable to those observed empirically using forces in the $x$ - and $y$-directions. In the simulations, the maximum rotation of the stiffness ellipse toward the $x$ - and $y$-axes was 11 and $24^{\circ}$, respectively. In the empirical study, the average change in orientation was $9.45^{\circ}$, with forces applied in the $x$-direction, and $21.35^{\circ}$, with forces applied in the $y$-direction. The changes observed empirically are thus comparable to those produced in simulations using commands that approximate the full range of control of muscle co-contraction.

The sensitivity of the orientation of the stiffness ellipse to the magnitude of the co-contraction command at the shoulder and elbow is shown in Fig. 4C. The darker regions on the upper mesh represent the empirically obtained range of stiffness associated with forces in $x$ and $y$ and in isotropic conditions (3rd day of training). These values are mapped onto the plane of shoulder and elbow co-contraction commands shown below. Based on this mapping, it is seen that subjects who trained with loads in the $y$-direction may have achieved changes in ellipse orientation primarily through elbow co-contraction (single joint elbow muscles and double joint muscles). For subjects in the $x$-direction, changes in orientation may have arisen mostly through shoulder co-contraction, but accompanied by co-contraction at the elbow.

The simulation results suggest that there is a limit to the angular change in the orientation of the stiffness ellipse even with high levels of elbow and shoulder co-contraction. To test this idea, we conducted a control study in which stiffness was measured following training using forces in the $x y$-direction, that is, roughly in the direction of minimum stiffness (see Fig. $1 A$ ). Our expectation was that any change in the orientation of the hand stiffness ellipse would be within the range observed with training in $x$ or $y$. Four new subjects trained over the course of 3 consecutive days. The task was identical to that described above except for the axis of load application. Over days, subjects tested in the $x y$-direction showed a progressive improvement in performance as indicated by the fact that the time outside of the target zone decreased systematically over the course of training $(P<0.01)$. The mean orientation of the stiffness ellipse also changed over days but reached a maximum $\left(17.8^{\circ}\right)$ that was comparable in magnitude to that observed for subjects tested with loads in the $y$-direction. Thus consistent with the results of the simulations, subjects were unable to change the orientation of the stiffness ellipse beyond that observed when loads were applied in $x$ or $y$ alone. 
A number of aspects of the statistical evaluation of the data require comment. The data presented here are angular measures and accordingly, since the range of variation is limited, potential violations of the assumption of an underlying Gaussian distribution need to be considered. We assessed the possibility of a truncated range by computing estimates of variation for measures of orientation. The SD of ellipse orientation averaged over days for forces in $x$ and $y$ and for isotropic loads was $5.6,7.6$, and $4.4^{\circ}$, respectively. Thus these data use only a small portion of the full angular range. We have in addition run tests for normality (Shapiro-Wilk $W$ ). Two tests were conducted, one using the orientation in the null-field before any learning and a second one on the residuals of the orientation observed in the force field (once the effect of group has been removed) on the final day of training. In neither case was a departure from normality found $(P>0.05)$.

The data on which impedance is estimated were collected over the course of learning. However, our analyses indicate that the changes that we have observed in stiffness occur primarily between experimental sessions rather than within a single day. Specifically, repeated measures ANOVA that included all three stiffness estimates per day revealed no reliable differences between estimates within a day for any of the directions of applied load $(P>0.05)$.

Displacements were delivered to the limb for purposes of stiffness estimation on $20 \%$ of trials. In general, it is desirable to deliver measurement displacements infrequently. However, in this context, it should be noted that increases in the frequency of measurement trials will tend to act against the development of directional changes in stiffness, since these particular displacements occur equally often in all directionsfavoring isotropic changes in the stiffness pattern. Since directional changes in stiffness are observed it would seem that the present measurement protocol if anything provides a conservative estimate of the magnitude of the directional effect.

\section{I S C U S S I O N}

It is known that arm impedance in postural maintenance is affected by musculoskeletal geometry, muscle mechanical behavior, reflex loops, and their gains and by level of cocontraction of antagonistic muscles. In this study, we have assessed the extent to which subjects can change the pattern of stiffness over the course of learning to resist destabilizing forces that are applied to the hand. We found that the orientation of the stiffness ellipse could be modified in an adaptive fashion, such that the change in orientation matched the direction of the externally applied load. We also used a physiologically realistic model of the arm to explore the manner in which control signals to muscles may be altered to reproduce the changes in orientation that we observed empirically. Our simulation studies suggest that modifications to the pattern of stiffness at the hand involve separate commands for coactivation at the shoulder and the elbow.

Support for the idea that the nervous system uses multiple co-contraction commands can be found in patterns of tonic EMG activity following voluntary movements. In particular, it has been shown that tonic activity in single-joint elbow muscles and bi-articular muscles varies with the magnitude of the preceding elbow movement and is not related to shoulder movement; tonic activity in single joint shoulder muscles varies with the magnitude of shoulder movement and is not related to elbow movement (Gribble and Ostry 1998). A similar relation is obtained between activity in muscles at the shoulder and the elbow and associated patterns of stiffness at these joints (Gomi and Osu 1998).

It may be noted that the simulated measures of joint impedance match those recorded empirically at the shoulder and elbow, which supports the idea that there are two separate commands for co-contraction-one that acts on single-joint shoulder muscles alone, and a second independent command that acts on the single-joint elbow and biarticular muscles. We have called this second command an "elbow" co-contraction command based on the fact that in this study this command appears to have an effect on elbow joint impedance and little effect on shoulder impedance, and the observations in the previous literature that in horizontal-plane tasks such as the one studied here, biarticular muscle activations tend to co-vary with elbow movement and single-joint elbow EMGs, and tend to be unrelated to shoulder movement and single-joint shoulder EMGs. It may be noted that for other anatomical configurations of the limb in three dimensions, biarticular muscles may become more related to shoulder motion, and co-contraction of biarticular muscles may have a greater effect on shoulder joint impedance. However, in this study, this appears not to be the case.

These findings may help to resolve a number of problems related to impedance control and muscle co-contraction. First of all, it has been difficult to obtain experimental evidence of voluntary changes to the pattern of impedance at the hand (Mussa-Ivaldi et al. 1985), and indeed the only evidence to date when the limb is stationary and net force is zero is limited to studies in which subjects were able to change stiffness based on visual feedback (Gomi and Osu 1998; Perreault et al. 2002). This study shows that impedance control develops gradually with practice and hence a possible reason for previous difficulties is that this ability is incompletely revealed with relatively small amounts of training. Furthermore, even with ample practice impedance change under static conditions is relatively small in magnitude, certainly far smaller than that seen during movement. Our simulations studies suggest that the changes observed here empirically are at a limit regardless of the level of co-contraction. Control studies that were specifically designed to test for the possibility of even greater changes to stiffness orientation were unable to produce a change in the orientation of the hand stiffness ellipse beyond that observed presently for loads acting in $x$ and $y$.

In this study, we have applied similar loads along the $x$ - and $y$-axes. Subjects in the $y$-direction showed a greater change in the orientation of the stiffness ellipse than subjects in $x$-direction. This asymmetry in the amount of orientation change is also found in the results of the simulation studies (Fig. 4A). Specifically, when co-contraction at the shoulder is increased while elbow co-contraction is held constant, the change in stiffness ellipse orientation reaches a plateau and changes little with further increases in co-contraction. In contrast, when elbow co-contraction is increased with co-contraction at the shoulder held constant, the stiffness ellipse orientation is observed to vary over a much larger range. The asymmetry in this pattern is presumably due to geometrical considerations since, at least in our modeling studies, our simulated commands equate for differences in the overall impedance of muscles acting at the shoulder and elbow. 
Gomi and Osu (1998) and Perreault et al. (2002) have used visual feedback to obtain voluntary changes to the orientation of the stiffness ellipse at the hand. The magnitude of change in both cases is similar to that reported here. Whereas in this study, the range of orientation change is $30.8^{\circ}\left(21.35^{\circ}\right.$ clockwise and $9.45^{\circ}$ counterclockwise), Perreault et al. report a range of $29.8^{\circ}$ when no forces are applied by the hand. This value decreases to about $18^{\circ}$ at $20 \%$ maximum voluntary contraction. An examination of Figs. 3 and 5 from Gomi and Osu suggests that a change in orientation of between 25 and $30^{\circ}$ is obtained with their procedure.

Stiffness ellipse size estimates in this study are larger than those reported by Gomi and Osu. Whereas we report values of 46,52 , and $135 \mathrm{~N} / \mathrm{cm}^{2}$ for loads in $x, y$, and under isotropic conditions, based on calibration bars provided in Figs. 3 and 5 of the Gomi and Osu (1998) paper, we estimate values of 15, 20 , and $55 \mathrm{~N} / \mathrm{cm}^{2}$ under comparable conditions. The difference in magnitude may be due to differences in the tasks and in particular to the availability of visual feedback in the Gomi and Osu procedure. Differences may also be due to the fact that our subjects held the handle of the manipulandum, whereas the subjects in the Gomi and Osu study did not—-their subjects had the arm supported by a plastic cuff. Perreault et al. (2002) do not provide measures of stiffness ellipse size. However the ellipses in their Fig. 4 are as much as twice the size of those in our study. In general, studies that use a handheld manipulandum report higher stiffness values regardless of the details of the task.

Recent studies by Milner and colleagues (Burdet et al. 2001; Franklin et al. 2003) merit comment. These researchers have shown that, during movements, subjects can modify the orientation of the stiffness ellipse at the hand to offset the effects of destabilizing loads. Impedance change during movement is large in comparison with that observed here in statics. In the small number of directions that have been studied during movement, the orientation of the hand stiffness ellipse can change by as much as about $45^{\circ}$, whereas when the limb is stationary, the changes even with extensive training are only one-half as large. Our modeling work suggests that, under static conditions, the changes we observe represent the full range of control under these conditions. One difference between static conditions and movement is the contribution to active muscle force that is associated with phasic muscle activity. Another possibility is that the range of stiffness control is actually larger during movement. These alternatives must be systematically assessed to understand the limits of stiffness control during movements and when the limb is in static equilibrium.

Milner (2002b) examined the relative roles of posture and joint stiffness in stabilizing hand position. As expected, subjects were more capable of stabilizing the hand when an external load acted in the direction of greatest stiffness than when the load was in the direction of least stiffness. Changes in posture had substantial effects on ability to stabilize the load. It may well be that subjects can circumvent limitations in the range of stiffness variation through changes in limb configuration.

We have used a physiological realistic arm model to obtain predictions of endpoint stiffness based on the use of different co-contraction commands. These same predictions could likely have been obtained by changing the values of terms in the joint stiffness matrix (Gomi and Osu 1998). However, the use of the full arm model is more informative in that it directly relates the control underlying individual muscles to patterns of simulated stiffness. The concern with using the joint stiffness matrix for this purpose is that there is no assurance that there is a simple mapping between the action of individual arm muscles and terms in the joint stiffness matrix. The stiffness matrix arises from a regression procedure in which any change in muscle activity might in principle affect any term in the matrix. Indeed Franklin and Milner (2003) have shown that all four elements of the joint stiffness matrix vary with both elbow and shoulder torque.

\section{A C K N O W LED G MENTS}

We thank G. Houle for technical assistance.

\section{G R A N T S}

This work was supported by the Ministry of Science, Research and Technology of Islamic Republic of Iran, the National Institute on Deafness and Other Communication Disorders, the Natural Sciences and Engineering Research Council of Canada, and Fonds Québécois de la Recherche sur la Nature et les Technologies.

\section{REFEREN CES}

Burdet E, Osu R, Franklin DW, Milner TE, and Kawato M. The central nervous system stabilizes unstable dynamics by learning optimal impedance. Nature 414: 446-449, 2001.

De Serres SJ and Milner TE. Wrist muscle activation patterns and stiffness associated with stable and unstable mechanical loads. Exp Brain Res 86: 451-458, 1991.

Feldman AG, Adamovich SV, Ostry DJ, and Flanagan JR. The origin of electromyograms - explanations based on the equilibrium point hypothesis. In: Multiple Muscle Systems: Biomechanics and Movement Organization, edited by Winters J and Woo S. New York: Spring-Verlag, 1990, p. 195-213.

Flash T and Mussa-Ivaldi FA. Human arm stiffness characteristics during the maintenance of posture. Exp Brain Res 82: 315-326, 1990.

Franklin DW, Burdet E, Osu R, Kawato M, and Milner TE. Functional significance of stiffness in adaptation of multijoint arm movements to stable and unstable dynamics. Exp Brain Res 151: 145-157, 2003.

Franklin DW and Milner TE. Adaptive control of stiffness to stabilize hand position with large loads. Exp Brain Res 152: 211-220, 2003.

Gomi H and Osu R. Task dependent viscoelasticity of human multijoint arm and its spatial characteristic for interaction with environment. J Neurosci 18: 8965-8978, 1998.

Gribble PL and Ostry DJ. Independent coactivation of shoulder and elbow muscles. Exp Brain Res 123: 355-360, 1998.

Gribble PL, Ostry DJ, Sanguineti V, and Laboissière R. Are complex control signals required for human arm movement? J Neurophysiol 79: 1409-1424, 1998.

Hogan N. The mechanics of multijoint posture and movement control. Biol Cybern 52: 315-331, 1985.

Lackner JR and DiZio P. Rapid adaptation to Coriolis force perturbations of arm trajectory. J Neurophysiol 72: 299-313, 1994.

Lacquaniti F, Carrozzo M, and Borghese NA. Time varying mechanical behavior of multijointed arm in man. J Neurophysiol 69: 1443-1464, 1993.

McIntyre J, Mussa-Ivaldi FA, and Bizzi E. The control of stable postures in the multi-joint arm. Exp Brain Res 110: 248-264, 1996.

Milner TE. Adaptation to destabilizing dynamics by means of muscle cocontraction. Exp Brain Res 143: 406-416, 2002a.

Milner TE. Contribution of geometry and joint stiffness to mechanical stability of the human arm. Exp Brain Res 143: 515-519, $2002 \mathrm{~b}$.

Mussa-Ivaldi FA, Hogan N, and Bizzi E. Neural, mechanical and geometric factors subserving arm posture in human. J Neurosci 5: 2732-2743, 1985.

Perreault EJ, Kirsch RF, and Crago PE. Voluntary control of static endpoint stiffness during force regulation tasks. J Neurophysiol 87: 2808-2816, 2002.

Sainburg RL, Ghez C, and Kalakanis D. Intersegmental dynamics are controlled by sequential anticipatory, error correction, and postural mechanisms. J Neurophysiol 81: 1045-1056, 1999.

Shadmehr R and Mussa-Ivaldi FA. Adaptive representation of dynamics during learning of a motor task. J Neurosci 14: 3208-3224, 1994.

Tsuji T, Morasso PG, Goto K, and Ito K. Human hand impedance characteristics during maintained posture. Biol Cybern 72: 475-485, 1995. 